\title{
IJCERT
}

International Journal of Computer Engineering in Research Trends Multidisciplinary, Open Access, Peer-Reviewed and fully refereed

\section{Enhanced MBFD Algorithm to Minimize Energy Consumption in Cloud}

\author{
Varun Jasuja $^{1^{*}}$, Dr. Rajesh Kumar Singh ${ }^{2}$ \\ $I^{*}$ Research Scholar, PTU, Jalandhar \\ ${ }^{2}$ Professor, SUS Institute of Computer, Tangori, Punjab \\ Email:Varunjasuja19@gmail.com,rajeshkripal@gmail.com
}

Available online at: $\underline{\text { http://www.ijcert.org }}$

Received: 01/02./2019,

Revised: 12/03/2019,

Accepted: 15/03/2019,

Published: 17/03/2019

\begin{abstract}
:-
Background/Objectives: Cloud computing is a shared pool of configurable computer system resources and higher-level services. These services quickly configured over the Internet to achieve consistency and economies of scale.

Methods/Statistical analysis: In this research, the DVFS (Dynamic Voltage and Frequency Scaling) mechanism is used to save energy in the cloud environment. In the existing work, MBFD has been used to check the resources in the physical machine. In case, if the resources are available, then the VM is placed over the PM. However, the problem is that the MBFD algorithm does not check the PM and hence result in higher energy consumption.

Findings: In this paper, the MBFD algorithm is enhanced by using the concept of DVFS along with the concept of location-aware algorithm. Due to this algorithm, VM which is near to the server is executed first by measuring the distance. To measure the performance the parameters such as energy consumption and TCJ are measured.
\end{abstract}

Improvements/Applications: The proposed framework reduced energy consumption and increased the total completed jobs.

Keywords: Cloud computing, DVFS, Virtualization, MBFD, Energy Consumption

\section{Introduction}

Cloud computing is a comparatively novel business model in the world of computing [1]. As per official NIST definition "Cloud computing is the model for permitting convenient, ubiquitous, ondemand network access towards sharing the pool of configurable computing resources like servers, networks, storage, services, and applications) that could be rapidly provisioned and released with least management effort or service provider interaction" $[2,3]$.

Figure 1 is defining cloud computing. The cloud computing is the transferring of computing services like storage, servers, databases, networking, analytics, and software $[4,5]$. The companies that offer these mentioned services are
Known as cloud providers and its usage charges cloud computing services.Below a few of the advantages of cloud computing that help cloud computing to be more reliable $[6,9]$ :

- Lower computer costs: In a cloud, a user does not need a high-powered (and, therefore high-priced) computer for running cloud computing web-based applications as applications run on the cloud, not on laptop or desktop PC. 


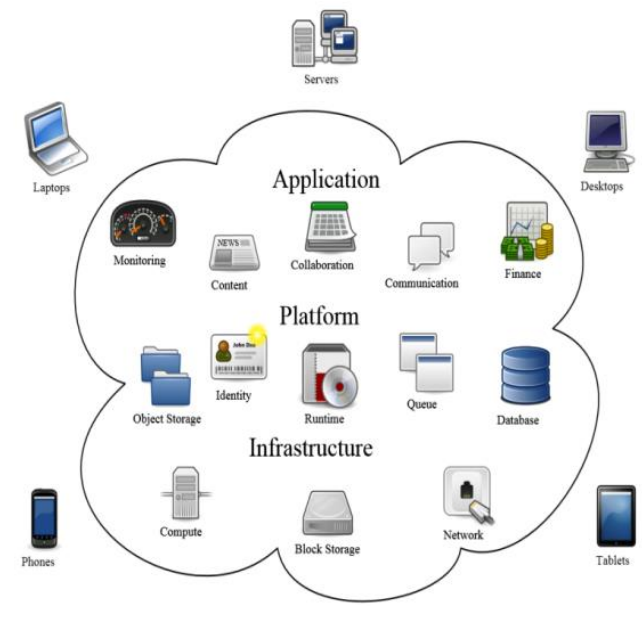

Figure 1. Cloud Computing

- Better performance: In cloud computing, the resources are utilized in an enhanced way. In cloud computing, the production has proposed a device on the reduction of performance concerns and knowing the relationship among SLA (Service level agreement) and performance. Variety of vendors has launched different cloud services that perform better as compared to existing ones using resource management, elastic computing power, and load balance and energy efficiency[7,8].

- Reduced software costs: In cloud computing, a user works on the model of pay per use means a user may only pay for some software by the period. There are some reasons for switching to cloud computing like less electricity, no capital cost, reduced staff cost, reduced operating cost with the elimination of downtime.

- Instant software updates: A new software-related advantage in cloud computing is that a user doesn't require to face obsolete software and high upgrade costs. In this context, next time when the user logged in to the cloud updates being shown automatically only if the app is web-based.

- Unlimited storage capacity: By storing more information in the cloud, a user may get unlimited space. Cloud provides $200 \mathrm{~GB}$ or more of storage capacity if needed.

- Universal document access: Cloud computing let the user access the applications with the documents from anyplace in the world and release the user from the limits of the desktop and provides group association $[10,25]$.

Green computing for reducing environmental waste and power consumption is required. The methods of green computing are Green Data Centre, Virtualization; Cloud computing, grid computing with Power Optimization. The main aim of green computing is intended for improving energy efficiency and reducing power consumption with the use of hazardous and toxic materials $[11,12,13]$. Green computing is used for designing the best computer system means that the speed of processing should be better which consume minimal power. It is the practice of manufacturing, designing, utilizing and discarding of computer server with few peripherals like printers, monitors, storage devices and so on, effectively and efficiently with no effect on the environment. However, these practices have effective server implementation and peripherals with the reduction of power consumption [14, 26].

The enhancement in the energy consumption amount in the world has taken as a critical problem. The variety of data centres has increased the energy demand for the maintenance and development of complex applications which is data-intensive nature. In the data centre management, the power consumption and application's QoS (Quality of Services) problem are vital for consideration $[16,17,18]$. The requirement for energy efficiency is now a critical factor for the designing of high-performance computing. Data centre needs a cooling system and more power in the cloud computing environment. There is a large amount of energy and power is used with the releasing of a large number of gases and heat. For reducing this power consumption, green computing is being used. Green computing is mainly concerned about reducing the total energy cost in the computation network. Some of the popular energy models in the cloud are the Random Energy Model, DVFS, and Radio Energy Model. DVFS is one of the most popular energy models for cloud architecture. Many researchers and Scientists have utilized it.

\section{DVFS (Dynamic Voltage and Frequency Scaling)}

DVFS is generally designed and intended for efficient energy management in the embedded system [19]. However, it could be considered as one of the effective methods for computation intensive server [27]. The authors have shown techniques that utilize DVFS take homogeneous PM (physical machine) and a slight interest in the concept of heterogeneity. This may be used in processing CPU tasks, but it is not appropriate for I/O intensive tasks as well as for memory. 


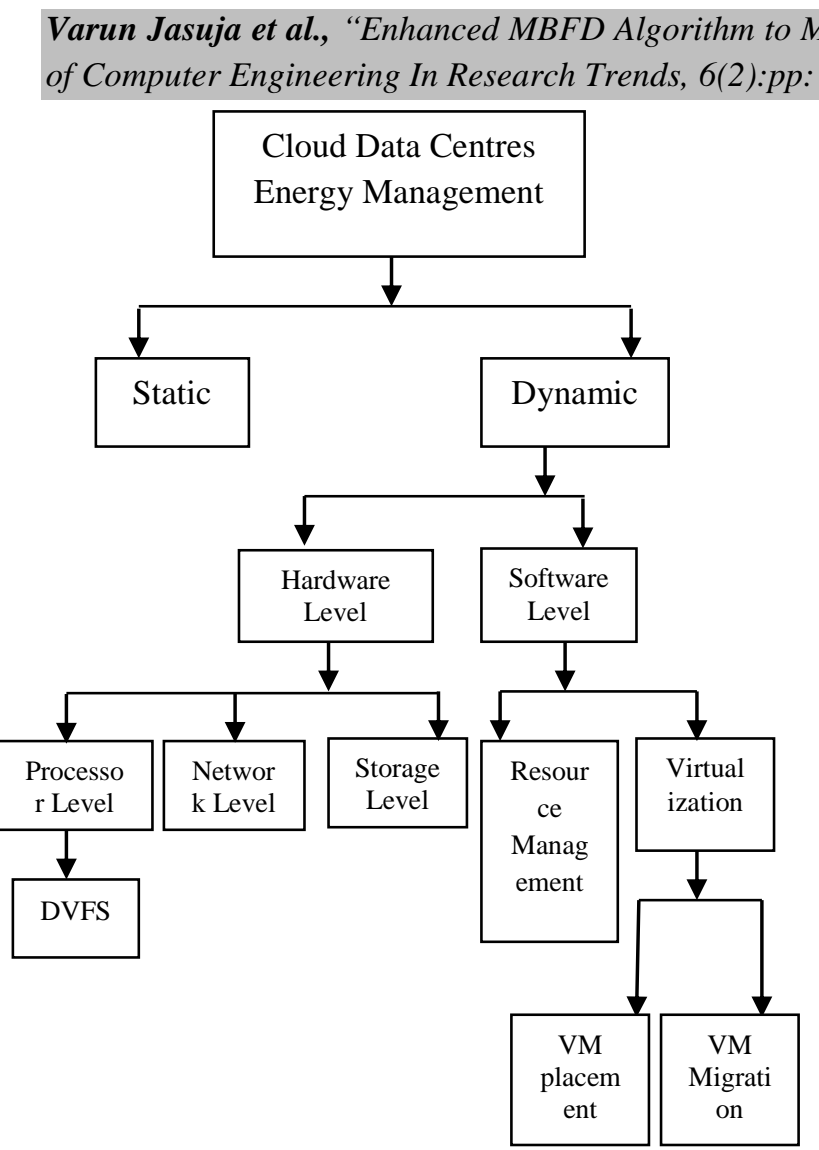

Figure 2. Energy Management Techniques Taxonomy

The energy model is applied over various models of the cloud-like network and storage level [20, 32]. A brief introduction of the models is as follows.

\section{- Network level}

The energy consumption of switch is dependent on the switch type, ports, employed caching solutions and port transmission rates [31].

\section{- Storage level}

Storage level consists of an essential fraction of the energy budget. The techniques at this level are defined below:

- Hardware-based technologies

- Disk Management techniques

- Caching technique

\section{Virtualization in Cloud Computing}

Virtualization is a knowledge that permits alone physical instance of some application or hardware resource to be shared among multiple organizations or tenants (customers) [28, 29, 30]. Virtualization helps cloud providers in creating different virtual machine instances on alone physical server [21,22,23]. Virtualization technology is using VM migration technology to improve cloud performance and optimize energy efficiency. Virtualization executes software layers among hardware and operating systems (and is called as VMMs (virtual machine monitors) or hypervisors) for controlling and mapping different VMs (Virtual machines) on a single platform [10,11]. The main tasks of VMM are resource allocation, hardware resource control, Operating System instruction conversion, Operating System processing, and Operating System interrupt handling.

\section{Problems With VM Handling And Proposed Solution}

VM placement requires resource utilization and resource availability [15]. As per the previously implemented algorithms like Anton and Buya, VM placement termed as MBFD checks the physical machine to have resources or not. If the resource is available then the VM is placed over the PM. This algorithm does not check the overloading of the PM, and hence this may lead to higher energy consumption [24]. The MBFD algorithm also does not consider mobile devices or availability on mobile devices. This paper focuses on developing an enhanced MBFD algorithm which also focuses on the location-aware services. The proposed algorithm integrates locationaware services along with the MBFD policy. The proposed algorithm also improves the allocation mechanism considering the load over the PM.

The traditional MBFD Algorithm is as follows

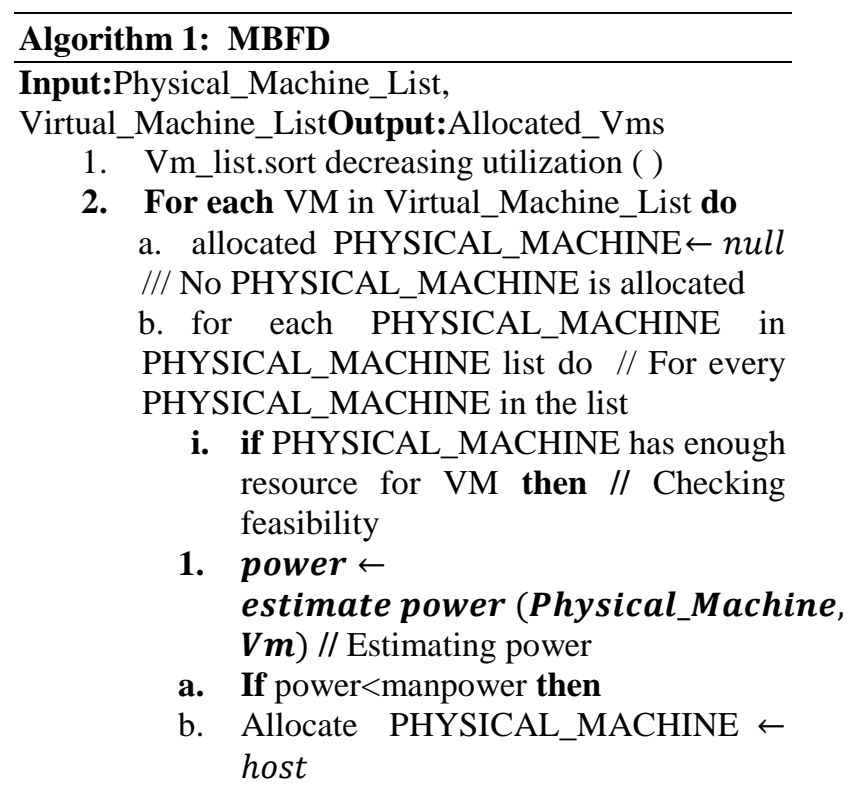


c. Mini power $\leftarrow$ power

3. if Allocated PHYSICAL_MACHINE $\neq$ null then

a. Allocate $\mathrm{VM}$ to allocated PHYSICAL_MACHINE

b. if Best_Utilization $=$ Max then

i. best VM_Value $\leftarrow$ VM break

ii. Hutil $\leftarrow$ Hutil - bestFitVM.getUtil()

iii. migration list.add (bestFitVM)

iv. VMList.remove (best VM_Value)

\section{End Algorithm}

The traditional MBFD algorithm checks the availability of the resources at the Physical Machine and distinguishes the VM as per their reducing capabilities of the CPU Utilization. Lowest CPU utilization VM is allocated first and, the process goes so on. The proposed algorithm utilizes the locationaware service of Mobile Cloud Servers. The VM which is near to the server is first evaluated with the distance measure. Algorithm 2 demonstrates the structure of the location value.

Algorithm 2: Find Suitable PM Based on Distance(PHYSICAL_MACHINE List, VM_Coordinates)

1. $V M_{X}=V M_{\text {Coordinates }} \cdot x$

2. $V M_{Y}=V M_{\text {Coordinates }} \cdot y$

3. For $_{\text {each }}$ PMI in Physical MachineList $_{\text {Pact }}$

a. Physical $_{\text {Machinex }}=P M I . X$

b. Physical $_{\text {Machiney }}=P M I . Y$

c. Location $_{\text {Value }}=$ [] Empty Location values

d. Location $_{\text {valuecount }}=0$;

e. $\quad$ dist $=\operatorname{sqrt}\left(\left(V M_{X}-\right.\right.$

Physical $\left._{\text {Machinex }}\right)^{2}+$

$\left.\left(V_{Y}-\text { Physical }_{\text {Machiney }}\right)^{2}\right)$

f. Evaluating the distance between the $V M$ and the PHYSICAL_MACHINE

g. IF dist $<V M_{\text {Coordinates }} *$ $25 \%$ range

i. Location value $\left[\right.$ Location $\left._{\text {valuecount }}\right]$ PMI

h. End if

ii. Location $_{\text {valuecount }}++$

4. End For

Algorithm 2 optimizes the MBFD algorithm by utilizing location-aware services. Algorithm 2 is implemented before Algorithm 1. The allocation policy is also enhanced utilizing Fuzzy Logic. The following rule set is applied.

Fuzzy logic takes three parameters as input. a) Load Value of Physical Machine

b) Queued Jobs

c) Energy Utilization

Output: future allocation possibility.

Three membership functions are applied for each parameter namely low, moderate and high.

Table 1. Fuzzy Structure

\begin{tabular}{|c|c|c|c|}
\hline $\begin{array}{c}\text { Load } \\
\text { Value }\end{array}$ & $\begin{array}{c}\text { Queued } \\
\text { Jobs }\end{array}$ & $\begin{array}{c}\text { Energy } \\
\text { Utilization }\end{array}$ & $\begin{array}{c}\text { Allocation } \\
\text { Possibility }\end{array}$ \\
\hline Low & Low & Low & High \\
\hline Low & Moderate & Low & High \\
\hline Low & Moderate & Low & Moderate \\
\hline Low & Low & Moderate & Moderate \\
\hline Low & Low & High & Moderate \\
\hline High & Low & Low & Moderate \\
\hline High & High & High & Low \\
\hline
\end{tabular}

Passing these values to the rule set will return the allocation possibility. This rule set is applied over the Algorithm 1 and 2. If the probability is moderate, then the allocation is avoided. If the allocation possibility is low then for sure the allowance is not made. Based on the presented architecture, Quality of Service (QoS) parameters is analyzed which is shown in the following section.

\section{Results and Analysis}

The evaluation of the proposed methodology is done utilizing Energy Consumption and Total Number of Completed Jobs (TCJ). The X-axis of each parameter analysis is Host to VM Ratio (HVR).

\section{Energy Consumption}

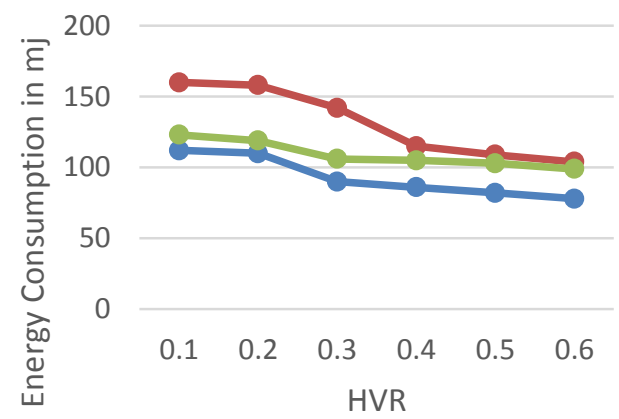

- Energy Consumption Proposed

- Energy Consumption MBFD

Energy Consumption MBFD + Location

Figure 3. Energy Consumption 
Figure 3 represents the Energy Consumption of the proposed framework and normal MBFD with MBFD associated with location architecture. The $\mathrm{x}$-axis is an HVR. If the number of VMs will increase then the load will be distributed, and hence less energy will be consumed but a still high amount of energy loss is observed from Figure 3 when only MBFD is applied. For an HVR value of .6, MBFD stands $104 \mathrm{mj}$ of energy consumption whereas when it is joined with location services, it reduces to $99 \mathrm{mj}$. The proposed architecture consumes the least amount of energy and stands a value of $78 \mathrm{mj}$ for the same scenario. Similarly, TCJ is also calculated and is illustrated in Figure 4.

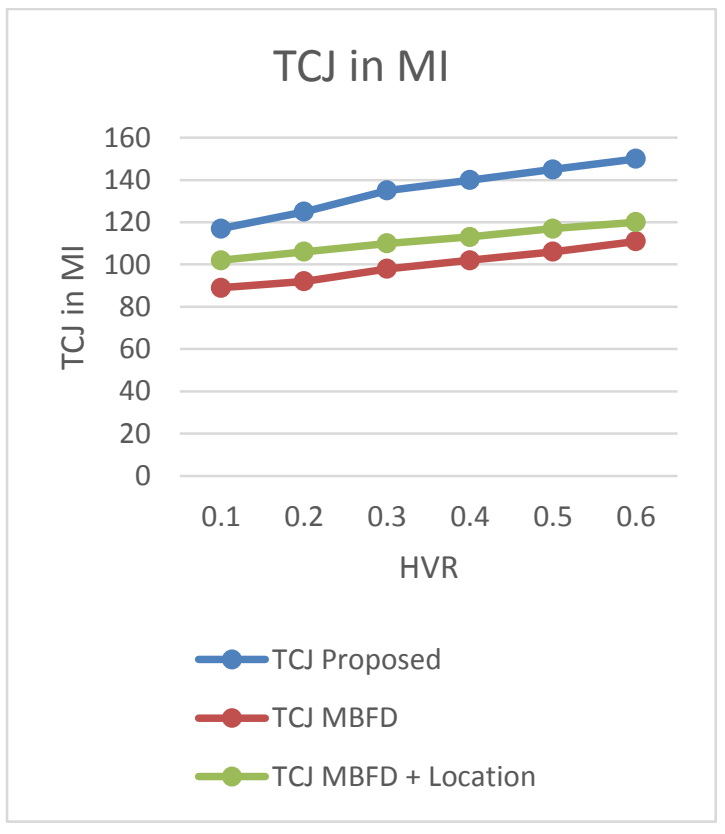

Figure 4. TCJ

TCJ of the proposed algorithm is 150 MI for .6 whereas $120 \mathrm{MI}$ for the location with MBFD and 111 MI for MBFD.

\section{Conclusion}

This paper presented an enhanced architecture of MBFD algorithm which integrates location-aware services into the Physical Machine framework. The presented structure considered the PMs as mobile and load structure is embedded into the MBFD architecture. A load balancer with the help of Fuzzy Logic is implemented. An enhanced rule set mechanism is presented and the evaluation is done on the base of Energy Consumption and Total jobs completed in Million Instruction. The proposed framework reduced energy consumption and increased total completed jobs. The proposed structure is also compared with MBFD and Integrated MBFD Location.

\section{References}

[1] P. Mell and T. Grance, "The NIST Definition of Cloud Computing", National Institute of Standards and Technology: U.S. Department of Commerce, NIST Special publication 800-145, September, 2011.

[2] Brown, Kevin, and Suresh Singh. "A network architecture for mobile computing." INFOCOM'96. Fifteenth Annual Joint Conference of the IEEE Computer Societies. Networking the Next Generation. Proceedings IEEE. Vol. 3. IEEE, 2002.

[3] Chen, $\mathrm{Xu}$, et al. "Efficient multi-user computation offloading for mobile-edge cloud computing." IEEE/ACM Transactions on Networking, Vol. 5, pp. 2795-2808, 2016.

[4] Dinh, Hoang T., et al. "A survey of mobile cloud computing: architecture, applications, and approaches." Wireless communications and mobile computing Vol. 13, Issue.18 pp.1587-1611, 2013.

[5] Gai, Keke, et al. "Dynamic energy-aware cloudlet-based mobile cloud computing model for green computing." Journal of Network and Computer Applications Vol. 59, pp. 46-54, 2016.

[6] Jo, Minho, et al. "Device-to-device-based heterogeneous radio access network architecture for mobile cloud computing." IEEE Wireless Communications Vol. 22, Issue. 3, pp. 50-58, 2015.

[7] Rahimi, M. Reza, et al. "Mobile cloud computing: A survey, state of the art and future directions." Mobile Networks and Applications Vol. 19, Issue.2, pp.133-143, 2014.

[8] Tong, Liang, Yong Li, and Wei Gao. "A hierarchical edge cloud architecture for mobile computing." INFOCOM 2016-The 35th Annual IEEE International Conference on Computer Communications, IEEE. IEEE, 2016.

[9] Yi, Shane, Cheng Li, and Qun Li. "A survey of fog computing: concepts, applications, and issues." Proceedings of the 2015 workshop on mobile big data. ACM, 2015.

[10] A. Beloglazov, and R. Buyya, "Energy Efficient Allocation of Virtual Machines in Cloud Data Centers", 10th IEEE/ACM International Conference on Cluster, Cloud and Grid Computing, pp. 577-578, 2010.

[11] Wei, B. (2012), "A novel energy optimized and workload adaptive modeling for live migration", International Journal of Machine Learning and Computing, Vol. 2, Issue. 2, pp. 158-162, 2012.

[12] Safari, Z., Bohlol, N., \&Fouladfar, E. (2015, April). Optimized live migration using NRU and modified clock policy. In e-Commerce in Developing Countries: With a focus on e-Business (ECDC), 2015 9th International Conference on (pp. 1-8). IEEE.

[13] A. Beloglazov, J. Abawajy, and R. Buyya, "Energy-aware Resource Allocation Heuristics for Efficient Management of Data Centers for Cloud Computing," Future Generation Computer Systems, Elsevier, Vol. 28, Issue 5, pp. 755-68, 2012.

[14] Chung, B. D., Jeon, H., \&Seo, K. K., "A framework of cloud service quality evaluation system-focusing on security quality evaluation," International Journal of Software Engineering Application, Vol. 8, Issue 4, pp. 4146, 2014 
[15] D. Jayasinghe, C. Pu, T. Eilam, M. Steinder, I. Whalley, and E. Snible, "Improving Performance and Availability of Services Hosted on IaaS Clouds with Structural Constraint-aware Virtual Machine Placement", IEEE International Conference on Services Computing, pp.7279, 2011.

[16] Ye, K., Jiang, X., Huang, D., Chen, J., \& Wang, B. Live migration of multiple virtual machines with resource reservation in cloud computing environments. In Cloud Computing (CLOUD), 2011 IEEE International Conference on IEEE, pp. 267-274, 2011.

[17] S. Esfandiarpoor, A. Pahlavan, and M. Goudarzi, "Virtual Machine Consolidation for Data center Energy Improvement", Cornell University Library, Ithaca, New York, 2013.

[18] Taha, A., Metzler, P., Trapero, R., Luna, J., \& Suri, N. ,Identifying and utilizing dependencies across cloud security services", In Proceedings of the 11th ACM on Asia Conference on Computer and Communications Security, ACM, pp. 329-340.

[19] L. Wu, SK Garg, and R. Buyya, "SLA-Based Resource Allocation for Software as a Service Provider (SaaS) In Cloud Computing Environments," 11th IEEE/ACM International Symposium on Cluster, Cloud And Grid Computing, pp. 195-204, 2011.

[20] Diallo, M. H., August, M., Hallman, R., Kline, M., Slayback, S. M., \& Graves, C.," AutoMigrate: a framework for developing intelligent, self-managing cloud services with maximum availability, Cluster Computing, Vol. 20, Issue. 3, pp. 1995-2012, 2017.

[21] Q. Zhang, Q. Zhu, and R. Boutaba, "Dynamic Resource Allocation For Spot Markets In Cloud Computing Environments," 4th IEEE International Conference on Utility and Cloud Computing, pp. 178-185, 2011.

[22] Kumar, M.," Review of practical issues of resource \& risk management in cloud computing", International Journal of Advanced Research in Engineering and Applied Sciences, Vol. 3, Issue. 5, pp. 23-34, 2014.

[23] S. Zaman, and D. Grosu, "A Combinatorial Auction-Based Mechanism for Dynamic VM Provisioning and Allocation in Clouds," IEEE Transactions on Cloud Computing, vol. 1, issue 2, pp.129-141, 2013.

[24] A. Quiroz, H. Kim, M. Parashar, N. Gnanasambandam, and N. Sharma, "Towards Autonomic Workload Provisioning for Enterprise Grids and Clouds", 10th IEEE/ACM International Conference on Grid Computing, Canada, pp. 50-57, 2009.

[25] Roy, N., Dubey, A., \&Gokhale, A. ,"Efficient autoscaling in the cloud using predictive models for workload forecasting", In Cloud Computing (CLOUD), 2011 IEEE International Conference on IEEE, pp. 500-507, 2011.

[26] Joao N. Silva, L. Veiga, and P. Ferreira, "Heuristic for Resources Allocation on Utility Computing Infrastructures",ACM Proceedings of the 6th International Workshop on Middleware for Grid Computing, 2008.

[27] Z. Xiao, W. Song, and Qi Chen, "Dynamic Resource Allocation Using Virtual Machines for Cloud Computing Environment", IEEE Transactions on Parallel and Distributed system, Vol. 24, Issue 6, pp. 1107-1117, 2013.

[28] Qiu, M., \&Sha, E. H. M. ," Cost minimization while satisfying hard/soft timing constraints for heterogeneous embedded systems", ACM Transactions on Design Automation of Electronic Systems (TODAES), Vol. 14, Issue. 2, pp. 25-32, 2009.
[29] Qiang Li, Q. Hao, L. Xiao, and Z. Li, “Adaptive Management of Virtualized Resources in Cloud Computing Using Feedback Control," IEEE First International Conference on Information Science and Engineering, pp. 99-102, 2009.

[30] Ahmed, M. T., \& Hussain, A., Survey on energy-efficient cloud computing systems.

[31] Esfandiarpoor, S., Pahlavan, A., \&Goudarzi, M. ,"Virtual Machine Consolidation for Datacenter Energy Improvement", 2013.

[32] Bertini, L., Leite, J. C., \&Mossé, D. ,"Power optimization for dynamic configuration in heterogeneous web server clusters", Journal of Systems and Software, Vol. 83, Issue. 4, pp. 585-598, 2010. 